\title{
Smart-802.11b MAC protocol for use with Smart Antennas
}

\author{
Harkirat Singh and Suresh Singh \\ Email: \{harkirat, singh\}@cs.pdx.edu \\ Department of Computer Science \\ Portland State University \\ Portland, OR 97207
}

\begin{abstract}
Smart antennas enable a receiver to determine the Direction of Arrival (DOA) of multiple transmissions as well as to form nulls in some number of directions to maximize SINR (Signal to Interference and Noise Ratio) of the received signal. We utilize the benefits of these capabilities to develop a simple modified version of the popular $802.11 b$ protocol. This protocol exhibits high throughput under a variety of network conditions and is fair. The performance of the protocol is examined exhaustively using joint simulation in OPNET and Matlab.
\end{abstract}

\section{INTRODUCTION}

Smart antennas (or adaptive array antennas) have some unique properties that enable us to achieve high throughputs in ad hoc network scenarios. A transmitter equipped with a smart antenna can form a directed beam towards its receiver and a receiver can similarly form a directed beam towards the sender, thus resulting in very high gain. A receiver can also identify the direction of multiple simultaneous transmitters by running DOA algorithms and use this information to determine the directions in which it should place the nulls. Placing nulls effectively cancels out the impact of interfering transmitters. In this paper we develop a simple $802.11 \mathrm{~b}$ based MAC protocol called Smart-802.11b that explicitly uses these three properties of smart antennas (beamforming, DOA, and nulling) to achieve high throughputs.

The two protocols developed in this paper are called SmartAloha([1], [2]) and Smart-802.11b, and, as the name implies, these two protocols are modifications to the well-known Aloha and $802.11 \mathrm{~b}$ protocols. In both cases, we have added functionality at the MAC layer to allow it to directly control the antenna: the MAC layer controls the direction of the beam and the direction of the nulls. In addition, the antenna provides the MAC layer with DOA information for all transmissions it can hear along with signal strength information. The main results of our paper are that our protocols show a very high throughput while maintaining fairness. The conclusion is that by appropriately exploiting the benefits of smart antennas, the capacity of wireless networks can be increased dramatically.

We use smart antenna model similar to our previous work [1], [2]. The remainder of this paper is organized as follows. In the next section we summarizes the previous work in this and related areas. In section III we describe the two

This work is funded by the NSF under grant ANIR-0125728. protocols called Smart-Aloha and Smart-802.11b. Section IV presents our OPNET-based simulation results and we provide a comparison with the omnidirectional case. We also analyze the fairness of our protocol in section IV-C. Finally, we summarize the main results in section $\mathrm{V}$.

\section{LITERATURE REVIEW}

Table I presents the main throughput results of the MAC protocols designed for directional antenna equipped nodes. It is important to note that, most of the MAC protocols summarized in the Table I do not fully exploit the nulling and beamforming capabilities of the smart antenna.

The salient features of our work are:

1) We use realistic antenna model, we develop liner array of antenna elements in Matlab and interface it with OPNET simulation.

2) We use nulling as well as DOA capabilities of the smart antennas.

3) We do not use additional channel for the tones.

4) Smart-802.11b does not use combination of omnidirectional/directional RTS and CTS as used by previous MAC protocols.

\section{DESCRIPTION OF THE PROTOCOLS}

Consider the case when a node $a$ needs to transmit a packet to node $b$ which is its one-hop neighbor. We assume that $a$ knows the angular direction of $b$ (as in [3]) and it can therefore form a beam in the direction of $b$. However, to maximize SINR, $b$ should also form a beam towards $a$ and form nulls in the direction of all other transmitters. In order to do this, $b$ needs to know two things - first, that $a$ is attempting to transmit to it, and second, the angular direction of all the other transmitters that interfere at $b$. The two protocols we discuss answer these two questions somewhat differently as we describe next.

\section{A. Smart-Aloha}

Smart-Aloha is a slightly modified version of the standard Slotted-Aloha protocol. To transmit a packet, a transmitter forms a beam towards its receiver and begins transmission. However, it prefaces its packet transmission with the transmission of a short (8-byte) pure tone (this is a simple sinusoid). 


\begin{tabular}{|c|c|c|c|c|c|c|c|}
\hline Prior Work & $\begin{array}{l}\text { Characteristics of } \\
\text { Simulation Expts. }\end{array}$ & \multicolumn{6}{|c|}{ Maximum Throughput } \\
\hline \multirow[t]{4}{*}{ [3] } & \multirow{4}{*}{$\begin{array}{l}\text { Switched beam antenna } \\
45^{0} \text { beamwidth, } 10 \mathrm{~dB} \text { gain, } 250 \mathrm{~m} \\
\text { range for omni, } 900 \mathrm{~m} \text { directional } \\
4 \mathrm{CBR} \text { sources, } 75 \mathrm{kbps}-2 \mathrm{Mbps} \text { each }\end{array}$} & \multicolumn{3}{|c|}{ Random Topology } & \multicolumn{3}{|c|}{ Mesh Topology } \\
\hline & & \multicolumn{6}{|c|}{$(\mathrm{N}=25,4$ hops $)$} \\
\hline & & MMAC & DMAC & 802.11 & MMAC & DMAC & 802.11 \\
\hline & & $\begin{array}{l}1000 \mathrm{kbps} \\
(5 \mathrm{x})\end{array}$ & $\begin{array}{l}400 \\
(2 \mathrm{x})\end{array}$ & $\begin{array}{l}200 \\
(1 \mathrm{x})\end{array}$ & $\begin{array}{l}800 \\
(4 x)\end{array}$ & $\begin{array}{c}300 \\
(1.5 \mathrm{x})\end{array}$ & $\begin{array}{l}200 \\
(1 \mathrm{x})\end{array}$ \\
\hline \multirow[t]{4}{*}[4]{} & \multirow{4}{*}{$\begin{array}{l}\text { Multi-beam antenna } \\
(1,2,4 \text { beams each) } \\
30^{0} \text { beamwidth, } 2 \mathrm{Mbps} \text { channel } \\
\text { slotted ( } 8 \mathrm{~ms} \text { slot), } 16 \mathrm{Kbit} \text { packet } \\
\text { (Throughput converted to } \\
\text { bps from pkts/slot/net) }\end{array}$} & \multicolumn{3}{|c|}{$\begin{array}{l}\text { Fully connected } \\
\text { (20 nodes })\end{array}$} & \multicolumn{3}{|c|}{$\begin{array}{l}\text { Multi-hop } \\
0 \text { nodes, } 5 \text { hops) }\end{array}$} \\
\hline & & 1 beam & 2 & 4 & 1 & 2 & 4 \\
\hline & & $12 \mathrm{Mbps}$ & 30 & 60 & 60 & 150 & 300 \\
\hline & & & & ax over $\mathrm{RO}$ & $\mathrm{A}, \mathrm{UxDN}$ & & \\
\hline \multirow[t]{3}{*}[5]{} & \multirow{3}{*}{$\begin{array}{l}\text { Adaptive antenna; 4x4, 8x8 } \\
\text { planar arrays, TDMA-802.11, 1-hop }\end{array}$} & \multicolumn{3}{|c|}{+2} & \multicolumn{3}{|c|}{$8 \times 8$} \\
\hline & & \multicolumn{6}{|c|}{ (55 nodes) } \\
\hline & & \multicolumn{3}{|c|}{8 pkts/packet time } & \multicolumn{3}{|c|}{9 packets/packet time } \\
\hline \multirow[t]{3}{*}{ [6] } & \multirow{3}{*}{$\begin{array}{l}\text { Switched beam } \\
60^{0} \text { beamwidth }\end{array}$} & \multicolumn{2}{|c|}{ Proposed } & \multicolumn{2}{|c|}{ "DRTS/DCTS } & \multicolumn{2}{|c|}{ CSMA/CA } \\
\hline & & \multicolumn{4}{|c|}{50 nodes) } & & \\
\hline & & \multicolumn{2}{|c|}{$3.5 \mathrm{Mbps}$} & \multicolumn{2}{|c|}{2.5} & \multicolumn{2}{|c|}{2} \\
\hline \multirow[t]{4}{*}{ [7] } & \multirow{4}{*}{$\begin{array}{l}\text { Circular adaptive antenna array } \\
\text { beamwidth } 64^{\circ}, 8 \mathrm{~dB} \text { gain }\end{array}$} & \multicolumn{3}{|c|}{25 nodes (grid) } & \multicolumn{3}{|c|}{225 nodes (grid) } \\
\hline & & No PC & Global PC & Local PC & No PC & Global PC & Local PC \\
\hline & & & & $(\mathrm{PC}-\mathrm{Pow}$ & Control) & & \\
\hline & & $1.3 \mathrm{x}$ & $1.7 \mathrm{x}$ & $2.1 \mathrm{x}$ & $2.6 \mathrm{x}$ & $4.75 x$ & $5.25 x$ \\
\hline [8] & $\begin{array}{l}\text { Ideal adaptive antenna } \\
20 \text { nodes, no nulling }\end{array}$ & $\begin{array}{r}P r c \\
\mathrm{O}-\mathrm{Omr}\end{array}$ & $\begin{array}{l}\text { col } \\
\text { irectional }\end{array}$ & & $\begin{array}{r}B e \\
20 \text { nodes }\end{array}$ & $\begin{array}{l}\text { vidth } \\
\text { gree }=7.5\end{array}$ & \\
\hline & & $\mathrm{D}-\mathrm{D}$ & ctional & $90^{\circ}$ & $60^{\circ}$ & $30^{\circ}$ & $10^{0}$ \\
\hline & (Improvement over omni case) & ORT & $\mathrm{CTS}$ & $35 \%$ & $57 \%$ & $100 \%$ & $142 \%$ \\
\hline & & DRT & $\mathrm{CTS}$ & $64 \%$ & $107 \%$ & $143 \%$ & $186 \%$ \\
\hline & Packet transmission is & DRT & $\mathrm{CTS}$ & $28 \%$ & $43 \%$ & $\mathrm{n} / \mathrm{a}$ & $57 \%$ \\
\hline & directional at sender/receiver & ORT & $\mathrm{CTS}$ & $29 \%$ & $50 \%$ & $86 \%$ & $121 \%$ \\
\hline & & & MA & $\mathrm{n} / \mathrm{a}$ & $400 \%$ & $\mathrm{n} / \mathrm{a}$ & $400 \%$ \\
\hline [9] & 6-element circular antenna array & & & (No $\mathrm{N}$ & ility) & & \\
\hline & (10 fixed patterns - no adaptation) & Omni & Rx dir & tional & DVCS & DVC & Ideal \\
\hline & $45^{0}$ beamwidth, 100 nodes, $1500 \mathrm{~m}^{2}$ & & Tx Omn & rectional & & Rx Directi & \\
\hline & 2-ray propagation model, no nulling & 400kbps & & & 1.4Mbps & & ps \\
\hline
\end{tabular}

TABLE I

SUMMARY OF DiRECTIONAL MAC PROTOCOL PERFORMANCE.



Fig. 1. False beamforming.

Idle nodes remain in an omni-directional mode and receive a complex sum of all such tones (note that the tones are identical for all nodes and thus we cannot identify the nodes based on the tone) and run a DOA algorithm to identify the direction and strength of the various signals. An idle node then beamforms in the direction of the maximum received signal strength and forms nulls in other directions and receives the transmitted packet. If the receiver node was the intended destination for the packet, it immediately sends an ACK using the already formed directed beam. On the other hand, if the packet was intended for some other node, then the receiver discards it. A sender waits for an ACK immediately after transmission of the packet and if it does not receive the ACK, it enters backoff in the standard way. Thus, the Smart-Aloha protocol follows a Tone/Packet/Ack sequence.

The intuition behind the receiver beamforming in the direction of the maximum signal is that, because of the directivity of the antenna, there is a high probability that it is the intended recipient for the packet. However, we note that in cases, as in Figure 1, the receiver $d$ incorrectly beamforms towards $a$ because $a$ 's signal is stronger than $b$ 's. While this is not a serious problem in most cases, we can envision scenarios where the $b \longrightarrow d$ transmission gets starved due to a large volume of $a \longrightarrow c$ traffic. An optimization we have therefore implemented is a single-entry cache scheme which works as follows:

- If a node beamforms incorrectly in a given timeslot, it remembers that direction in a single-entry cache.

- In the next slot, if the maximum signal strength is again in the direction recorded in the single-entry cache, then the node ignores that direction and beamforms towards the second strongest signal.

- If the node receives a packet correctly (i.e., it was the intended recipient), it does not change the cache.

- If it receives a packet incorrectly, it updates the cache with this new direction.

- If there is no packet in a slot from the direction recorded in the cache, the cache is reset. 
This simple mechanism ensures that in cases similar to Figure 1 , connections are not starved. However, we can construct more complex scenarios where a single-entry cache will fail to prevent starvation. In these cases, more sophisticated multipleentry caching schemes are required. However, in our simulations, we only use the single-entry caching scheme because the probability of more complex scenarios resulting in starvation are very rare.

\section{B. Smart-802.11b}

The second protocol we have developed, Smart-802.11b, is based on the $802.11 \mathrm{~b}$ standard with some changes as noted below. As in the case of the Smart-Aloha protocol, transmitters beamform towards their receivers and transmit a short sendertone to initiate communication. However, unlike Smart-Aloha, the transmitter does not immediately follow the tone with a packet. Instead, it waits for a receiver-tone and only then transmits its packet. After transmission of a packet, it waits for the receipt of an ACK. If there is no ACK, it enters backoff as in $802.11 \mathrm{~b}$. Figure 2 provides a state diagram of our tonebased protocol. The behavior of the protocol in various states can be summarized as follows:

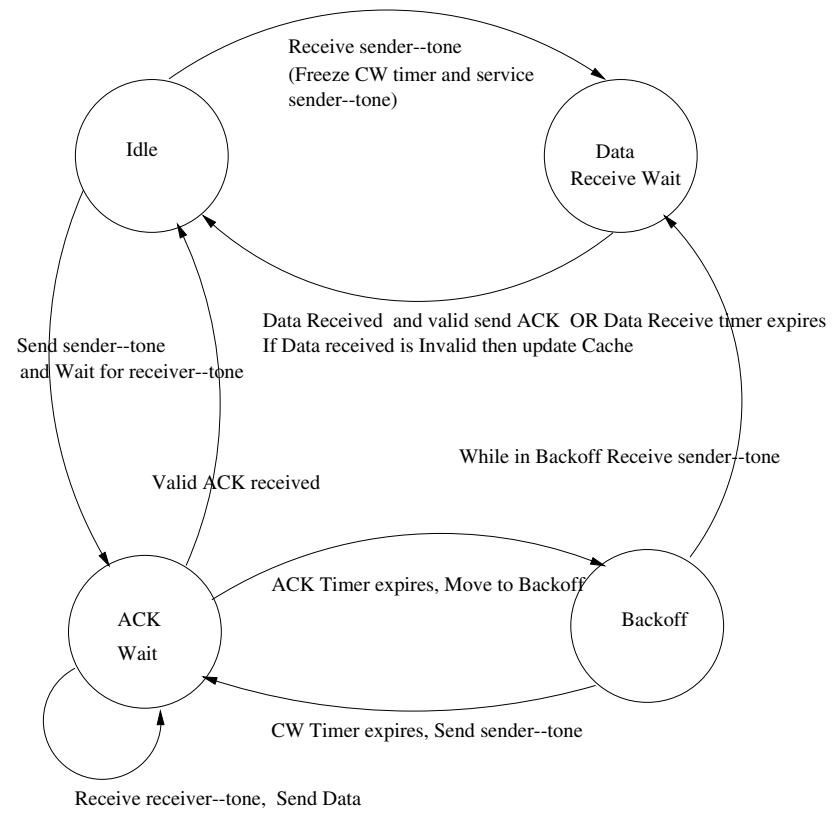

Fig. 2. State diagram of the Smart-802.11b protocol.

Idle: In case a node has no packet to send, it will remain in the Idle state and set its antenna to operate in the omni-directional mode. If it receives a sendertone from some other node, it will move into the Data Receive Wait state. On the other hand, if it wishes to send data, it will beamform in the direction of the receiver. It chooses a random number between $[0 . . \mathrm{CW}]$ and sets the $\mathrm{CW}$ (Contention Window) timer ${ }^{1}$. When the $\mathrm{CW}$ timer expires, it sends a sender-tone in the direction of the receiver and moves to the ACK Wait state. If, before the $\mathrm{CW}$ timer expires, the node receives a sender-tone from

\footnotetext{
${ }^{1}$ The random number selected is multiplied with $20 \mu$ sec.
}

another node, it will freeze its $\mathrm{CW}$ timer and move to Data Receive wait state.

Data Receive Wait: A node will move to this state in the event it receives a sender-tone. The node will beamform towards the sender and then randomly defer transmitting the receiver-tone by choosing a random waiting period between $[0 . .32] \times 20 \mu \mathrm{sec}$. The reason for deferring the reply is to minimize the chance of several receiver-tones colliding at the sender ${ }^{2}$.

After transmitting a receiver-tone, the node remains in this state for $2 \tau$ (twice the maximum propagation delay+tone transmission time). If it does not hear a transmission, it returns to the Idle state. If it hears the start of a transmission, it remains in this state and receives the packet. It then discards the packet if the packet was meant for some other node If, however, the packet was meant for it, then it sends an ACK.

Ack Wait: If the sender node receives a receiver-tone before the tone RTT timer goes off (which is twice the tone transmission time plus propagation delay) it will transmit the data packet. Reception of a valid ACK will move the node to the idle state, and if packets are there in the queue then it will schedule the one at the head of the queue. The node will move to Backoff state under two conditions 1) a receiver-tone did not arrive, 2) an ACK was not received following transmission of the data packet.

Backoff: The node computes a random Backoff interval (as in 802.11) and remains in backoff for this time period (it also resets its antenna to omni-directional mode). If, however, a sender-tone is received, it freezes the backoff timer and enters the Data Receive Wait state. If the node is in backoff, upon expiration of the timer, it retransmits the sender-tone, increments the retransmit counter, and enters the ACK Wait state. A packet is discarded after the retransmit counter exceeds Max_Retransmit $=7$, as in the IEEE 802.11 standard.

The reception of a data packet by a node may be interfered with transmissions of sender-tones, receiver-tones, or other data packets (since our protocol does not take care of hidden terminals). A node engaged in receiving a data packet can dynamically form nulls towards new interferers, but this process takes some time (we model this time as the length of a sender-tone). Thus, the data packet will have errors due to this interference. We combat this error by relying on FEC (Forward Error Correcting) codes as used in IEEE 802.11e, where $(224,208)$ shortened Reed Solomon (RS) codes are used. In 802.11e, a MAC packet is split into blocks of 208 octets and each block is separately coded using a RS encoder. A $(48,32) \mathrm{RS}$ code, which is also a shortened RS code, is used for the MAC header, and CRC-32 is used for the Frame Check Sequence (FCS). Note that any RS block can correct up to 8 octet errors. 


\begin{tabular}{|l|l|}
\hline \multicolumn{2}{|c|}{ Simulation Parameters } \\
\hline Background Noise + ambient Noise & $-143 \mathrm{~dB}$ \\
Propagation model & Free space \\
Bandwidth & $1,000 \mathrm{kHz}$ \\
Min frequency & $2,402 \mathrm{MHz}$ \\
Data Rate & $2000 \mathrm{kbps}$ \\
Carrier Sensing Threshold & $+3 \mathrm{~dB}$ \\
Minimum SINR & $9 \mathrm{~dB}$ \\
Bit Error & Based on BPSK \\
& Modulation curve \\
Maximum radio range & $250 \mathrm{~m}$ \\
Packet Size & $16 \mathrm{~KB}$ \\
Simulation time & $200 \mathrm{sec}$ \\
\hline \multicolumn{2}{|c|}{ Single Hop } \\
\hline Number of nodes & 20 \\
Area & $100 \times 100 \mathrm{~m}$ \\
\hline \multicolumn{2}{|c|}{ Multihop } \\
\hline Number of nodes \\
Area
\end{tabular}

TABLE II

OPNET SIMULATION PARAMETERS.

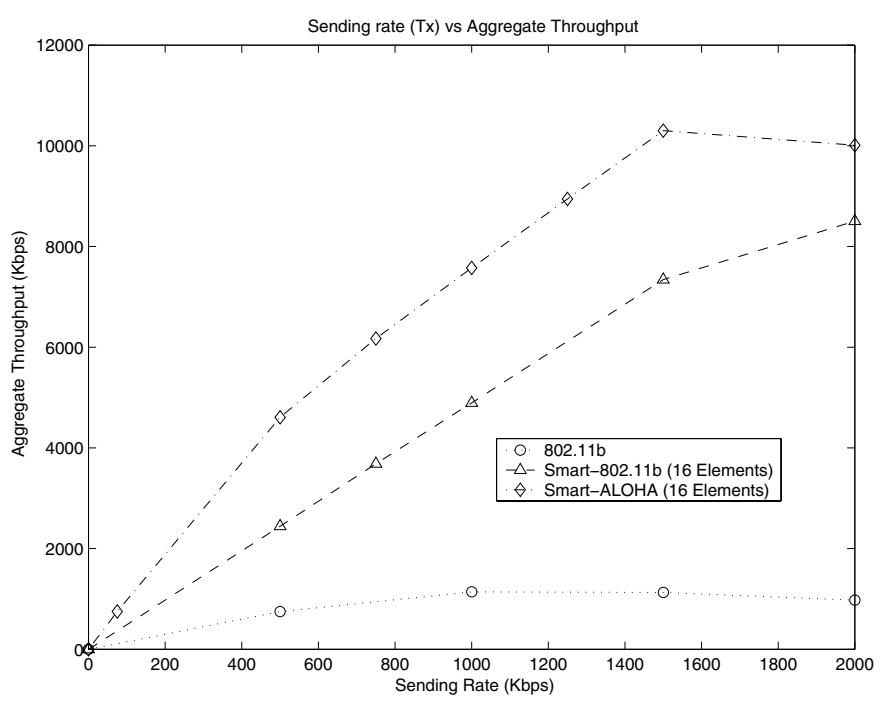

Fig. 3. Single-hop case with 20 nodes.

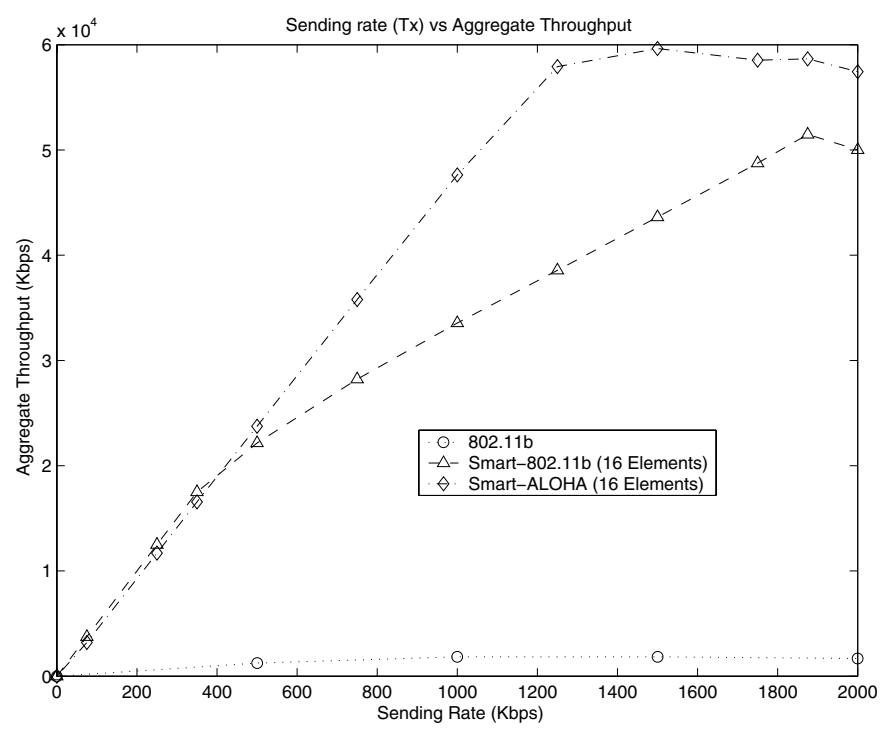

Fig. 4. Five-hop case with 100 nodes.

\section{Fairness of Smart-Aloha and Smart-802.11b}

We performed a study of the fairness properties of the two new protocols and $802.11 \mathrm{~b}$ presented here using prior work [10], [11], [12] as a guide. Since our goal was to examine the fairness of the MAC protocol (as opposed to the fairness of TCP flows), we considered the single hop flows illustrated in Figure 5. The dotted lines between two nodes in the figures indicates that the two nodes can hear one another. The arrows indicate the direction of flows and we used 2Mbit/sec CBR traffic for each flow with 512 byte packets. The maximum channel capacity is also $2 \mathrm{Mbit} / \mathrm{sec}$ and the remaining parameters were set as per Table II.

Table III shows the data rate achieved by each flow in each of the three topologies from Figure 5. In Topology 1, nodes 1 and 2 are within range of one another and node 1 is in fact in the second symmetric lobe formed by node 2 towards node 3. In the case of Smart-802.11b, this causes node 2 to
${ }^{2}$ Note that our tones do not carry information about the sender and the receiver so if all the nodes who receive a sender-tone (and are in idle state) respond immediately then the sender will detect a collision. 

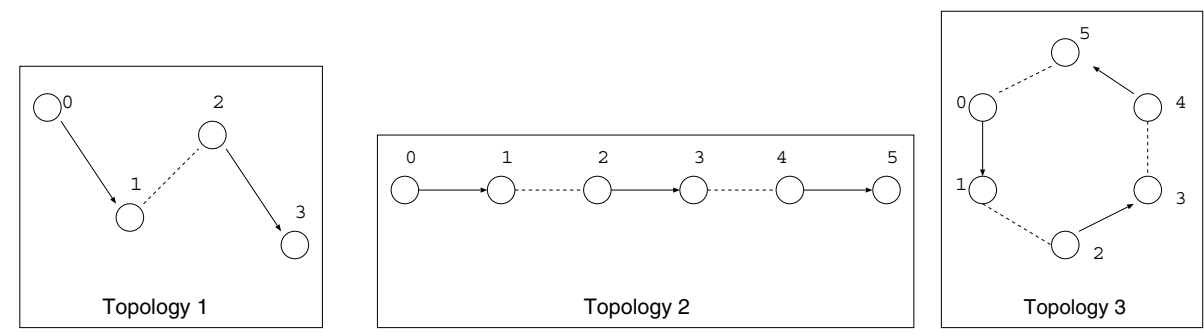

Fig. 5. Topologies used for fairness study.

\begin{tabular}{|c|c|c|c|}
\hline \multicolumn{5}{|c|}{ Topology 1 } \\
\hline Flow & $\begin{array}{c}802.11 b \\
(\mathrm{Mbps})\end{array}$ & $\begin{array}{c}\text { Smart-802.11b } \\
(\mathrm{Mbps})\end{array}$ & $\begin{array}{c}\text { Smart-Aloha } \\
(\mathrm{Mbps})\end{array}$ \\
\hline $0 \longrightarrow 1$ & 0.661 & 0.919 & 1.957 \\
$2 \longrightarrow 3$ & 0.663 & 1.282 & 1.978 \\
\hline \hline \multicolumn{4}{|c|}{ Topology 2 } \\
\hline Flow & $802.11 b$ & Smart-802.11b & Smart-Aloha \\
$0 \longrightarrow 1$ & 0.089 & 0.871 & 1.958 \\
$2 \longrightarrow 3$ & 0.108 & 0.908 & 1.827 \\
$4 \longrightarrow 5$ & 0.567 & 0.860 & 1.931 \\
\hline \hline \multicolumn{4}{|c|}{ Topology 3 } \\
\hline Flow & $802.11 b$ & Smart-802.11b & Smart-Aloha \\
$0 \longrightarrow 1$ & 0.427 & 0.745 & 1.573 \\
$2 \longrightarrow 3$ & 0.433 & 0.914 & 1.459 \\
$4 \longrightarrow 5$ & 0.430 & 0.924 & 1.896 \\
\hline
\end{tabular}

TABLE III

AVERAGE DATA RATES OF DIFFERENT FLOWS.

sometimes incorrectly send a receiver-tone to node 2 resulting in lower throughput for the $0 \longrightarrow 1$ flow. Smart-Aloha is not affected by this because node 1 is closer to node 0 and forms a bean towards 0 while forming a null towards 2 using the DOA information. In the case of Topology 2, the three flows are equally sharing the channel because, unlike in Topology 1 , the second lobe of a transmitter (such as node 2) does not face unintended receivers (node 1). Finally, due to the symmetry of Topology 3, all the flows are equally affected by the second lobe and thus exhibit similar throughputs. In addition to the topologies discussed above, we studied other topologies including the star topology with four transmitters sending to one common receiver (as in [12]). We note that all the flows shared the channel equally in this case as well.

\section{CONCLUSION}

This paper presents two simple tone-based protocols for use with smart antenna systems. These protocols do not explicitly combat hidden terminals yet they show very high throughput, exceeding that of many other protocols. We also demonstrate that our protocols share the channel fairly among multiple competing flows. In many ways our approach here is contrary to the current trend of designing increasingly complex MAC protocols for directional antenna systems. The overall conclusion is that smart antennas can indeed be used to great benefit in ad hoc networks and can enhance the performance of the popular slotted-aloha and $802.11 \mathrm{~b}$ protocols.

\section{ACKNOWLEDGMENTS}

We would like to thank OPNET for their technical support.

\section{REFERENCES}

[1] Harkirat Singh and Suresh Singh, "Doa-aloha: Slotted aloha for ad hoc networking using smart antennas," in IEEE VTC Fall'03, 6 - 9 Oct 2003.

[2] Harkirat Singh and Suresh Singh, "A mac protocol based on adaptive beamforming for ad hoc networks," in IEEE Pimrc'03, 7 - 10 Sep 2003.

[3] Romit Roy Choudhury, Xue Yang, Ram Ramanathan, and Nitin H. Vaidya, "Using directional antennas for medium access control in ad hoc networks," in ACM/SIGMOBILE MobiCom 2002, 23 - 28 Sep 2002.

[4] Lichun Bao and J.J. Garcia-Luna-Aceves, "Transmission scheduling in ad hoc networks with directional antennas," in ACM/SIGMOBILE MobiCom 2002, 23 - 28 Sep 2002.

[5] S. Bellofiore, J. Foutz, R. Govindarajula, I. Bahceci, C. A. Balanis, A. S. Spanias, J.M. Capone, and T. M. Duman, "Smart antenna system analysis, integration, and performance for mobile ad-hoc networks (manets)," IEEE Transaction on Antennas and Propagation, vol. 50, no. 5 , pp. $571-581$, May 2002.

[6] T. ElBatt and B. Ryu, "On the channel reservation schemes for ad hoc networks utilizing directional antennas," in WPMC'02, 2002.

[7] N. Fahmy, T. D. Todd, and V. Kezys, "Ad hoc networks with smart antennas using 802.11-based protocols," in IEEE ICC'02.

[8] G. Marvin Sanchez, "Multiple access protocols with smart antennas n multihop ad hoc rural-area networks," M.S. thesis, Royal Institute of Technology, Sweeden, Radio Communication Systems Laboratory, Department of Signals, Sensors and Sytems, June 2002.

[9] Rajiv Bagrodia Mineo Takai, Jay Martin and Aifeng Ren, "Directional virtual carrier sensing for directional antennas in mobile ad hoc networks," in ACM/SIGMOBILE MobiHoc 2002, Oct 2002.

[10] T. Nandagopal, T-E. Kim, X. Gao, and V. Bharghavan, "Achieving mac layer fairness in wireless packet networks," in ACM MOBICOM'OO, August 2000, pp. 87 - 98 .

[11] B. Bensaou, Y. Wang, and C. C. Ko, "Fair medium access in 802.11 based wireless ad-hoc networks," in ACM MOBIHOC'00, August 2000, pp. $99-106$.

[12] V. Bharghavan, A. Demers, S. Shenker, and L. Zhang, "Macaw: A media access protocol for wireless lans," in ACM SIGCOMM'94, August 1994, pp. $212-225$. 